Electronic Supporting Information for

\title{
Novel Glassy Tetra(N-alkyl-3-bromocarbazole-6-yl)silanes as Building Blocks for Efficient and Nonaggregating Blue-Light-Emitting Tetrahedral Materials
}

Xue-Ming Liu, ${ }^{1, *}$ Jianwei $\mathrm{Xu},{ }^{1} \mathrm{Xuehong} \mathrm{Lu}^{2}$ and Chaobin $\mathrm{He}^{1, *}$

${ }^{1}$ Institute of Materials Research and Engineering, 3 Research Link, Singapore 117602

${ }^{2}$ School of Materials Science and Engineering, Nanyang Technological University

* Corresponding authors

Xue-Ming Liu (Dr.) and Chaobin He (Dr.)

Institute of Materials Research \& Engineering, 3 Research Link, Singapore 117602

Fax: 65-68727528

Email addresses: xm-liu@,imre.a-star.edu.sg; cb-he@,imre.a-star.edu.sg

\section{Contents}

Part I. Experimental procedures and characterization data on newly synthesized compounds and polymers.

Part II. Table and spectroscopic information for newly synthesized compounds and polymers. 


\section{Part I. Experimental procedures and characterization data on newly synthesized compounds and polymers.}

General Information: Unless stated otherwise, all reagents and solvents were of commercial grade, and used as received. All reactions were performed under a purified nitrogen atmosphere using the standard Schlenk technique. Tetrahydrofuran (THF) was distilled over $\mathrm{CaH}_{2}$ before use. The ${ }^{1} \mathrm{H}$ NMR spectra were recorded at $25{ }^{\circ} \mathrm{C}$ on a Bruker AVANCE 400 spectrometer. Mass spectra were recorded on a Bruker Autoflex TOF/TOF MALDI-TOF MS spectrometer using dithranol/Ag-TFA as a matrix. The molecular weights of polymers were determined by gel permeation chromatography (GPC) using polystyrenes as standards and THF as a mobile phase at $1.0 \mathrm{~mL} /$ minute. UV-Vis spectra were measured with a UV-Vis spectrometer (Shimadzu, UV2501 PC) at $25^{\circ} \mathrm{C}$. Fluorescence spectra were recorded on a LS50B Luminescence Spectrometer (Perkin Elmer) at $25{ }^{\circ} \mathrm{C}$. Glass transition temperatures were determined by differential scanning calorimetry (DSC) experiments using a TA 2920 Modulated DSC instrument with a ramp speed of $10{ }^{\circ} \mathrm{C} / \mathrm{min}$. Thermogravimetric analyses (TGA) were conducted on a Perkin Elmer thermogravimetric Analyzer TGA 7 under a heating rate of $20{ }^{\circ} \mathrm{C} / \mathrm{min}$ and a nitrogen flow rate of $20 \mathrm{~cm}^{3} / \mathrm{min}$. WAXS measurements were conducted using Bruker X-ray diffractometer using $\mathrm{Cu}$ $\mathrm{K} \alpha(\lambda=1.541 \AA)$ radiation. Thin films of the samples prepared by a hydraulic press die were used for the measurements. The X-ray tube was operated at $40 \mathrm{kV}$ and $40 \mathrm{~mA}$. Elemental analyses were performed by the Elemental Analysis Laboratory of the Department of Chemistry at the National University of Singapore. 
The starting compounds N-alkyl-3,6-dibromocarbazole (alkyl = ethyl, $n$-butyl and $n$-hexyl), ${ }^{1}$ 9,9-dihexylfluorene-2-boronic acid and 9,9-dihexylfluorene-2,7-bis(trimethylene boronate) ${ }^{2}$ were prepared according to the literature methods.

Quantum yield determination. The photoluminescent quantum yields ( $\left.\Phi_{\mathrm{PL}}\right)$ of the compounds in THF were determined using a solution of quinine sulfate as a standard $\left(c a .1 \times 10^{-5} \mathrm{M}\right.$ in 0.1 $\mathrm{M} \mathrm{H}_{2} \mathrm{SO}_{4}$, having a quantum yield of 55\%). Dilute sample solutions were used for the determinations (absorbance $<0.1$ ). All samples were degassed before measurements. The correction of emission spectra was carried out by reference to quinine bisulfate in acidic solution (400-600 nm) according to the literature method. ${ }^{3}$ The $\Phi_{\mathrm{PL}}$ values of films were determined using 9,10-diphenylanthracene as a standard (dispersed in PMMA films with a concentration lower than $1 \times 10^{-3} \mathrm{M}$ and a quantum efficiency of $83 \%$ ). Thin films (absorbance $<0.2$ ) were dried at $50{ }^{\circ} \mathrm{C}$ under a vacuum oven for $16 \mathrm{~h}$ before measurements. Values are calculated according to Equation (1), where $\Phi_{\text {unk }}$ is the fluorescence quantum yield of the sample, $\Phi_{\text {std }}$ is the fluorescence quantum yield of the standard, $I_{\text {unk }}$ and $I_{\text {std }}$ are the integrated emission intensities of the sample and the standard, respectively, $A_{\text {unk }}$ and $A_{\text {std }}$ are the absorbances of the sample and the standard at the excitation wavelength, respectively, and $\eta_{\text {unk }}$ and $\eta_{\text {std }}$ are the refractive indexes of the corresponding solutions (pure solvents were assumed). ${ }^{4}$

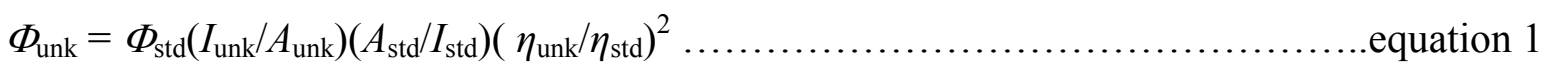

\section{A general procedure for the synthesis of precursors 1-3 by the Grignard method. N-hexyl-}

3,6-dibromocarbazole $(10 \mathrm{~g}, 24.4 \mathrm{mmol})$ and $\mathrm{Mg}(0.62 \mathrm{~g}, 25.5 \mathrm{mmol})$ were dissolved in THF $(150 \mathrm{~mL})$. The reaction was initiated by addition of $\mathrm{I}_{2}$. The reaction mixture was stirred at $r$. t. for 
$2 \mathrm{~h}$, and then refluxed overnight. The obtained Grignard was cooled to r. t. and was added $\mathrm{Si}(\mathrm{OMe})_{4}(1.03 \mathrm{~g}, 6.8 \mathrm{mmol})$ in one portion. The reaction mixture was stirred at r. t. for 2 days, and then refluxed for $4 \mathrm{~h}$. The above reaction solution was cooled to r. t. and was added $30 \mathrm{~mL}$ $1 \mathrm{~N} \mathrm{HCl}$, and then stirred for $0.5 \mathrm{~h}$. The organic layer was isolated, aqueous layer was extracted with dichloromethane $(2 \times 100 \mathrm{~mL})$. The combined organic layer was washed with $10 \% \mathrm{NaOH}$, brine, water, and then dried $\left(\mathrm{MgSO}_{4}\right)$. The organic solution was filtered through celite to give a clear yellow solution. The crude product was purified by silica gel column chromatography (chloroform $/ n$-hexane 1:1). After separation of the small compounds 1-3, the polymer residue in column was washed out with ethyl acetate, and was further purified by continuous extraction with acetone using Soxhlet apparatus for $24 \mathrm{~h}$.

A general procedure for the synthesis of precursors 1-3 by mono-lithiation. In a $250 \mathrm{~mL} 2$ necked RBF was added N-hexyl-3,6-dibromocarbozole (6 g, $14.7 \mathrm{mmol})$ and $100 \mathrm{~mL}$ freshly dried THF. The solution was cooled to $-78^{\circ} \mathrm{C}$ using dry ice/acetone bath. To the cooled solution was added $n$-BuLi $(1.6 \mathrm{M}, 11 \mathrm{~mL}, 17.6 \mathrm{mmol})$ dropwise through a syringe. The reaction mixture was allowed to stir at $-78{ }^{\circ} \mathrm{C}$ for another two hours, and then was added $\mathrm{Si}(\mathrm{OMe})_{4}(0.66 \mathrm{~mL}, 4.4$ mmol) in one portion. The reaction mixture was stirred at $-78{ }^{\circ} \mathrm{C}$ for $4 \mathrm{~h}$, and then was allowed to warm to room temperature and stirred for another 5 days. After that, the reaction mixture was refluxed for another $4 \mathrm{~h}$. Upon completion, solvent was removed. The residue was re-dissolved in chloroform $(100 \mathrm{~mL})$, and was washed with water and dried $\left(\mathrm{MgSO}_{4}\right)$. The chloroform solution was filtered through celite and concentrated to give a yellow solid. The crude product was subjected to column chromatography (chloroform $/ n$-hexane 1:1) to give compounds $\mathbf{1 - 3}$. After separation of the small compounds 1-3, the polymer residue in column was washed out 
with ethyl acetate, and was further purified by continuous extraction with acetone using Soxhlet apparatus for $24 \mathrm{~h}$.

Tetra(N-ethyl-3-bromocarbazole-6-yl)silane, 1. A white solid. Anal. Calcd. for $\mathrm{C}_{56} \mathrm{H}_{44} \mathrm{Br}_{4} \mathrm{~N}_{4} \mathrm{Si}: 1120.698 . \mathrm{C}, 59.90 ; \mathrm{H}, 4.00 ; \mathrm{Br}, 28.52 ; \mathrm{N}, 5.00$; found $\mathrm{C}, 59.92 ; \mathrm{H}, 4.46 ; \mathrm{Br}$, 28.16; N, 4.56. ${ }^{1} \mathrm{H}$ NMR $\left(\mathrm{CDCl}_{3}\right): \delta 1.45\left(\mathrm{t},{ }^{3} J_{\mathrm{H}-\mathrm{H}}=7.2 \mathrm{~Hz}, 12 \mathrm{H}\right), 4.38\left(\mathrm{q},{ }^{3} J_{\mathrm{H}-\mathrm{H}}=7.2 \mathrm{~Hz}, 8 \mathrm{H}\right)$, $7.30\left(\mathrm{~d},{ }^{3} J_{\mathrm{H}-\mathrm{H}}=8.4 \mathrm{~Hz}, 4 \mathrm{H}\right), 7.47\left(\mathrm{~d},{ }^{3} J_{\mathrm{H}-\mathrm{H}}=8.0 \mathrm{~Hz}, 4 \mathrm{H}\right), 7.53\left(\mathrm{dd},{ }^{3} J_{\mathrm{H}-\mathrm{H}}=8.4 \mathrm{~Hz},{ }^{4} J_{\mathrm{H}-\mathrm{H}}=1.8\right.$ $\mathrm{Hz}, 4 \mathrm{H}), 7.86\left(\mathrm{~d},{ }^{3} J_{\mathrm{H}-\mathrm{H}}=8.0 \mathrm{~Hz}, 4 \mathrm{H}\right), 8.17\left(\mathrm{~d},{ }^{4} J_{\mathrm{H}-\mathrm{H}}=1.8 \mathrm{~Hz}, 4 \mathrm{H}\right), 8.43(\mathrm{~s}, 4 \mathrm{H}) .{ }^{13} \mathrm{C} \mathrm{NMR}$ $\left(\mathrm{CDCl}_{3}\right): \delta 14.2,38.1,108.9,110.2,112.2,122.3,123.7,125.0,125.1,128.6,128.8,133.7$ 138.9, 141.8. MALDI-TOF MS (m/z): $1146.4\left(\mathrm{M}^{+}+\mathrm{Na}^{+}\right), 1222.2\left(\mathrm{M}^{+}+\mathrm{Ag}^{+}\right)$.

Tetra[N-(n-butyl)-3-bromocarbazole-6-yl)silane], 2. A white solid. Anal. Calcd. for $\mathrm{C}_{64} \mathrm{H}_{60} \mathrm{Br}_{4} \mathrm{~N}_{4} \mathrm{Si}:$ 1232.914. C, 62.35; H, 4.91; Br, 25.92; N, 4.54. Found: $\mathrm{C}, 62.12 ; \mathrm{H}, 5.14 ; \mathrm{Br}$, 25.54; N, 4.36. ${ }^{1} \mathrm{H}$ NMR $\left(\mathrm{CDCl}_{3}\right): \delta 0.96\left(\mathrm{t},{ }^{3} J_{\mathrm{H}-\mathrm{H}}=7.2 \mathrm{~Hz}, 12 \mathrm{H}\right), 1.42(\mathrm{~m}, 8 \mathrm{H}), 1.87(\mathrm{~m}, 8 \mathrm{H})$, $4.30\left(\mathrm{t},{ }^{3} J_{\mathrm{H}-\mathrm{H}}=7.2 \mathrm{~Hz}, 8 \mathrm{H}\right), 7.29\left(\mathrm{~d},{ }^{3} J_{\mathrm{H}-\mathrm{H}}=8.8 \mathrm{~Hz}, 4 \mathrm{H}\right), 7.46\left(\mathrm{~d},{ }^{3} J_{\mathrm{H}-\mathrm{H}}=8.0 \mathrm{~Hz}, 4 \mathrm{H}\right), 7.52(\mathrm{dd}$, $\left.{ }^{3} J_{\mathrm{H}-\mathrm{H}}=8.8 \mathrm{~Hz},{ }^{4} J_{\mathrm{H}-\mathrm{H}}=1.8 \mathrm{~Hz}, 4 \mathrm{H}\right), 7.84\left(\mathrm{~d},{ }^{3} J_{\mathrm{H}-\mathrm{H}}=8.0 \mathrm{~Hz}, 4 \mathrm{H}\right), 8.15\left(\mathrm{~d},{ }^{4} J_{\mathrm{H}-\mathrm{H}}=1.8 \mathrm{~Hz}, 4 \mathrm{H}\right)$, 8.41 (s, $4 \mathrm{H}) .{ }^{13} \mathrm{C} \mathrm{NMR}\left(\mathrm{CDCl}_{3}\right): \delta 14.2,20.9,31.5,43.4,109.1,110.5,112.2,122.2,123.6$, 125.0, 128.6, 128.7, 133.7, 139.5, 142.3. MALDI-TOF MS (m/z): $1230.6\left(\mathrm{M}^{+}\right), 1258.5\left(\mathrm{M}^{+}+\right.$ $\left.\mathrm{Na}{ }^{+}\right), 1338.5\left(\mathrm{M}^{+}+\mathrm{Ag}^{+}\right)$.

Tetra[N-(n-hexyl)-3-bromocarbazole-6-yl)silane], 3. A white solid. Anal. Calcd. for $\mathrm{C}_{72} \mathrm{H}_{76} \mathrm{Br}_{4} \mathrm{~N}_{4} \mathrm{Si}:$ 1345.13. C, 64.29; H, 5.70; $\mathrm{Br}, 23.76 ; \mathrm{N}, 4.17$. Found: $\mathrm{C}, 64.00 ; \mathrm{H}, 5.33 ; \mathrm{Br}$, 23.69; N, 4.21. ${ }^{1} \mathrm{H} \mathrm{NMR}\left(\mathrm{CDCl}_{3}\right): \delta 0.87\left(\mathrm{t},{ }^{3} J_{\mathrm{H}-\mathrm{H}}=6.8 \mathrm{~Hz}, 12 \mathrm{H}\right), 1.31(\mathrm{~m}, 16 \mathrm{H}), 1.34(\mathrm{~m}, 8 \mathrm{H})$, $1.87(\mathrm{~m}, 8 \mathrm{H}), 4.30\left(\mathrm{t},{ }^{3} J_{\mathrm{H}-\mathrm{H}}=6.8 \mathrm{~Hz}, 8 \mathrm{H}\right), 7.29\left(\mathrm{~d},{ }^{3} J_{\mathrm{H}-\mathrm{H}}=8.8 \mathrm{~Hz}, 4 \mathrm{H}\right), 7.47\left(\mathrm{~d},{ }^{3} J_{\mathrm{H}-\mathrm{H}}=8.0 \mathrm{~Hz}\right.$, $4 \mathrm{H}), 7.53\left(\mathrm{dd},{ }^{3} J_{\mathrm{H}-\mathrm{H}}=8.8 \mathrm{~Hz},{ }^{4} J_{\mathrm{H}-\mathrm{H}}=2.0 \mathrm{~Hz}, 4 \mathrm{H}\right), 7.84\left(\mathrm{~d},{ }^{3} J_{\mathrm{H}-\mathrm{H}}=8.0 \mathrm{~Hz}, 4 \mathrm{H}\right), 8.16\left(\mathrm{~d},{ }^{4} J_{\mathrm{H}-\mathrm{H}}=\right.$ 
$2.0 \mathrm{~Hz}, 4 \mathrm{H}), 8.41(\mathrm{~s}, 4 \mathrm{H}) .{ }^{13} \mathrm{C} \mathrm{NMR}\left(\mathrm{CDCl}_{3}\right): \delta 14.4,22.9,27.3,29.3,31.9,43.6,109.2,110.5$, $112.2,122.2,123.6,124.4,125.0,128.6,128.7,133.6,139.5,142.3$. MALDI-TOF MS (m/z): $1352.6\left(\mathrm{M}^{+}+7\right), 1374.4\left(\mathrm{M}^{+}+\mathrm{Na}^{+}\right), 1488.6\left(\mathrm{M}^{+}+\mathrm{Ag}^{+}\right)$.

P1. A gray powder. ${ }^{1} \mathrm{H}$ NMR $\left(\mathrm{CDCl}_{3}\right): \delta 1.46$ (br.), 4.38 (br.), 6.8-8.8 (m) ${ }^{13} \mathrm{C} \mathrm{NMR}\left(\mathrm{CDCl}_{3}\right): \delta$ 14.1, 38.0, 108.6, 108.8, 110.2, 112.2, 119.3, 120.9, 122.2, 123.3, 123.7, 125.1, 125.9, 128.3, $128.6,128.8,133.1,133.8,138.9,141.7$.

P2. A gray powder. ${ }^{1} \mathrm{H}$ NMR ( $\mathrm{CDCl}_{3}$ ): $\delta 0.94$ (br.), 1.30 (br.), 1.82 (br.), 4.23 (br.), 7.0-8.8 (m).

${ }^{13} \mathrm{C}$ NMR $\left(\mathrm{CDCl}_{3}\right): \delta 14.2,20.8,31.4,43.2,108.8,109.0,110.4,112.1,119.0,120.7,120.9$, $122.1,123.2,123.6,124.9,125.9,126.7,128.1,128.4,128.6,132.7,133.3,139.4,142.1$.

P3. A gray powder. ${ }^{1} \mathrm{H}$ NMR ( $\left.\mathrm{CDCl}_{3}\right): \delta 0.83$ (br.), 1.24 (br.), 1.69 (br.), 4.06 (br.), 6.9-8.7 (m). ${ }^{13} \mathrm{C}$ NMR ( $\mathrm{CDCl}_{3}$ ): $\delta 14.3,22.8,27.2,29.2,31.8,43.3,108.7$ (br.), 110.3 (br.), 111.7 (br.), 119.1 (br.), 120.6 (br.), 121.7 (br.), 123.2 (br.), 124.8 (br.), 126.5 (br.), 128.0 (br.), 132.8 (br.), 139.1 (br.), 141.9 (br.).

Synthesis of tetra\{[(9,9-dihexyl-3-fluorenyl)-N-hexyl]carbazole-6-yl\}silane, 4. Compound 3 (0.4 g, $0.30 \mathrm{mmol}), 9,9$-dihexylfluorene-2-boronic acid (0.55 g, $1.45 \mathrm{mmol}),\left[\mathrm{Pd}\left(\mathrm{PPh}_{3}\right)_{4}\right](20 \mathrm{mg}$, $1 \mathrm{~mol} \%)$ were dissolved in toluene $(6 \mathrm{~mL})$ and $2 \mathrm{M}$ aq. $\mathrm{K}_{2} \mathrm{CO}_{3}(4 \mathrm{~mL})$. The reaction mixture was degassed by bubbling with $\mathrm{N}_{2}$ for 15 minutes, and then was heated at $90-100{ }^{\circ} \mathrm{C}$ for 2 days. Upon completion, the organic layer was separated, and was washed with water and dried $\left(\mathrm{MgSO}_{4}\right)$. The product was isolated by silica gel column chromatography (chloroform $/ n$-hexnae $1: 2, \mathrm{R}_{\mathrm{f}}=$ $0.25)$ and was obtained as a white solid in $60 \%$ yield $(0.42 \mathrm{~g})$. Anal. Calcd. for $\mathrm{C}_{172} \mathrm{H}_{208} \mathrm{~N}_{4} \mathrm{Si}$ : 2359.67. C, 87.55; H, 8.89; N, 2.37. Found: C, 87.10; $\mathrm{H}, 8.97 ; \mathrm{N}, 2.37 .{ }^{1} \mathrm{H} \mathrm{NMR}\left(\mathrm{CDCl}_{3}\right): \delta 0.72$ $\left(\mathrm{t},{ }^{3} J_{\mathrm{H}-\mathrm{H}}=7.0 \mathrm{~Hz}, 24 \mathrm{H}\right), 0.87\left(\mathrm{t},{ }^{3} J_{\mathrm{H}-\mathrm{H}}=6.8 \mathrm{~Hz}, 12 \mathrm{H}\right), 1.03(\mathrm{~m}, 64 \mathrm{H}), 1.33(\mathrm{~m}, 16 \mathrm{H}), 1.45(\mathrm{~m}, 8$ $\mathrm{H}), 1.93\left(\mathrm{t},{ }^{3} J_{\mathrm{H}-\mathrm{H}}=7.2 \mathrm{~Hz}, 8 \mathrm{H}\right), 2.00(\mathrm{~m}, 16 \mathrm{H}), 4.36\left(\mathrm{t},{ }^{3} J_{\mathrm{H}-\mathrm{H}}=7.2 \mathrm{~Hz}, 8 \mathrm{H}\right), 7.29\left(\mathrm{~d},{ }^{3} J_{\mathrm{H}-\mathrm{H}}=7.6\right.$ 
$\mathrm{Hz}, 4 \mathrm{H}), 7.32\left(\mathrm{dd},{ }^{3} J_{\mathrm{H}-\mathrm{H}}={ }^{3} J_{\mathrm{H}-\mathrm{H}}{ }^{\prime}=7.6 \mathrm{~Hz}, 8 \mathrm{H}\right), 7.50\left(\mathrm{~d},{ }^{3} J_{\mathrm{H}-\mathrm{H}}=8.8 \mathrm{~Hz}, 8 \mathrm{H}\right), 7.64-7.74(\mathrm{~m}, 16$

H), $7.79\left(\mathrm{~d},{ }^{3} J_{\mathrm{H}-\mathrm{H}}=8.4 \mathrm{~Hz}, 4 \mathrm{H}\right), 7.88\left(\mathrm{~d},{ }^{3} J_{\mathrm{H}-\mathrm{H}}=8.4 \mathrm{~Hz}, 4 \mathrm{H}\right), 8.40(\mathrm{~s}, 4 \mathrm{H}), 8.68(\mathrm{~s}, 4 \mathrm{H}) .{ }^{13} \mathrm{C}$ $\operatorname{NMR}\left(\mathrm{CDCl}_{3}\right): \delta 14.3,22.9,24.1,27.4,29.4,30.0,31.8,31.9,40.8,43.7,55.5,109.0,109.2$ $119.4,119.9,120.2,121.9,123.2,123.5,123.9,124.3,125.7,126.4,127.0,128.6,133.5,139.9$ 140.3, 141.3, 141.4, 142.6, 151.3, 151.7. MALDI-TOF MS (m/z): $2355.0\left(\mathrm{M}^{+}\right.$- 4).

Synthesis of polymer 5. Compound $3\left(\begin{array}{llll}0.4 & \mathrm{~g} & 0.3 & \mathrm{mmol}\end{array}\right)$, 9,9-dihexylfluorene-2,7bis(trimethylene boronate) $(0.3 \mathrm{~g}, 0.6 \mathrm{mmol}),\left[\mathrm{Pd}\left(\mathrm{PPh}_{3}\right)_{4}\right](14 \mathrm{mg}, 1 \mathrm{~mol} \%)$ were dissolved in toluene $(20 \mathrm{~mL})$ and $2 \mathrm{M}$ aq. $\mathrm{K}_{2} \mathrm{CO}_{3}(14 \mathrm{~mL})$. The reaction mixture was degassed by bubbling with $\mathrm{N}_{2}$ for 15 minutes, and then was heated at $90-100{ }^{\circ} \mathrm{C}$ for 2 days. Upon completion, the organic layer was separated, and was washed with water and dried $\left(\mathrm{MgSO}_{4}\right)$. After removal of the organic solvent, the solid was continuously extracted with acetone for $48 \mathrm{~h}$ using Soxhlet apparatus (the catalyst residue, unreacted monomers and low molecular oligomers were dissolved in acetone). The remaining solid residue was then extracted with THF for $24 \mathrm{~h}$. The THF solution was concentrated to give the soluble polymer 5 in $38 \%$ yield $(0.2 \mathrm{~g})$ as a yellow solid. Anal. Calcd. for $\left(\mathrm{C}_{122} \mathrm{H}_{140} \mathrm{~N}_{4} \mathrm{Si} \bullet 4 \mathrm{H}_{2} \mathrm{O}\right)_{\mathrm{n}}$ : $(1762.636)_{\mathrm{n}}$. C, 83.13; H, 8.46; N, 3.18. Found: C, 83.22; H, 8.66; N, 3.38; Br, 1.92 (from residual end groups). ${ }^{1} \mathrm{H}$ NMR $\left(\mathrm{CDCl}_{3}\right): \delta 0.72$ (br., 12 H), 0.87 (br., 12 H), 1.03 (br., 32 H), 1.32 (br., 24 H), 1.93 (br., 8 H), 2.05 (br., 8 H), 4.36 (br., 8 H), 7.50 (br., 12 H), 7.65 (br., 20 H), 7.79 (br., 4 H), 7.86 (br., 4 H), 8.41 (br., 4 H), 8.70 (br., 4 H). ${ }^{13} \mathrm{C}$ NMR $\left(\mathrm{CDCl}_{3}\right): \delta 14.4,21.5,22.9,24.1,27.3,29.4,30.0,30.7,31.8,31.9,34.6,40.8$, 43.6, 55.6, 109.0, 109.2, 119.4, 120.1, 121.9, 123.2, 123.5, 123.8, 124.3, 125.9, 126.4, 127.0, $127.2,128.6,129.2,133.4,136.2,139.8,140.3,141.0,142.6,152.0$. 


\section{References:}

(1) Li, Y.; Ding, J.; Day, M.; Tao, Y.; Lu, J.; D’iorio, M. Chem. Mater. 2004, 16, 2165.

(2) Liu, B.; Yu, W.-L.; Lai, Y.-H.; Huang, W. Macromolecules 2000, 33, 8945.

(3) Eaton, D. F. Pure Appl. Chem. 1988, 60, 1107.

(4) Joshi, H. S.; Jamshidi, R.; Tor, Y. Angew. Chem. Int. Ed. 1999, 38, 2721.

Part II. Table and spectroscopic information for newly synthesized compounds and polymers.

Table S1. Synthesis, thermal and optical properties of precursors $\mathbf{1 - 3}{ }^{a}$

\begin{tabular}{|c|c|c|c|c|c|c|c|c|}
\hline & \multicolumn{2}{|c|}{ yield $(\%)$} & \multirow{2}{*}{$\begin{array}{c}\text { Mw } \\
(\mathrm{Mw} / \mathrm{Mn})\end{array}$} & \multirow[b]{2}{*}{$T_{g}^{c}\left({ }^{\circ} \mathrm{C}\right)$} & \multirow[b]{2}{*}{$T_{d}^{d}\left({ }^{\circ} \mathrm{C}\right)$} & \multicolumn{2}{|c|}{ abs. } & \multirow{2}{*}{$\begin{array}{c}\mathrm{em}^{e} \\
\lambda_{\max }(\mathrm{nm}) \\
(\mathrm{fwhm} / \mathrm{nm})\end{array}$} \\
\hline & conditions $\mathrm{i}^{b}$ & conditions ii $^{b}$ & & & & $\lambda_{\max }(\mathrm{nm})$ & $\log \varepsilon_{\max }$ & \\
\hline 1 & 8 & 43 & ----- & 137 & 250 & $277,304,340,356$ & $\begin{array}{c}5.15,4.78,4.17 \\
4.15\end{array}$ & $\begin{array}{c}378,394 \\
(49)\end{array}$ \\
\hline P1 & 42 & 12 & $\begin{array}{l}3820 \\
(1.1)\end{array}$ & 135 & 379 & $276,301,334$ & ------ & $414(78)$ \\
\hline 2 & 9 & 41 & ------ & 96 & 368 & $277,304,340,356$ & $\begin{array}{c}5.14,4.75,4.18 \\
4.16\end{array}$ & $\begin{array}{c}377,394 \\
(46)\end{array}$ \\
\hline P2 & 40 & 10 & $\begin{array}{l}5520 \\
(1.5)\end{array}$ & 119 & 358 & $278,302,335$ & ------ & $417(69)$ \\
\hline 3 & 9 & 41 & ----- & 65 & 434 & $278,304,340,356$ & $\begin{array}{c}5.18,4.78,4.20 \\
4.20\end{array}$ & $379(47)$ \\
\hline P3 & 38 & 11 & $\begin{array}{l}7880 \\
(1.8)\end{array}$ & 109 & 403 & $278,303,339$ & ----- & $395(67)$ \\
\hline
\end{tabular}

${ }^{a}$ Sample concentrations for 1-3 were $1.75-2.23 \times 10^{-5} \mathrm{M}$ and for P1-P3 were $1.0 \times 10^{-2} \mathrm{~g} / \mathrm{L} .{ }^{b}$ Conditions $\mathrm{i}$ and ii refer to those described in Scheme $1 .{ }^{c}$ The middle temperatures; ${ }^{d} T_{d}$ is defined as the temperature at which a $5 \%$ weight loss is recorded by the TGA analysis. ${ }^{e}$ The excitation wavelengths were $301-304 \mathrm{~nm}$. 


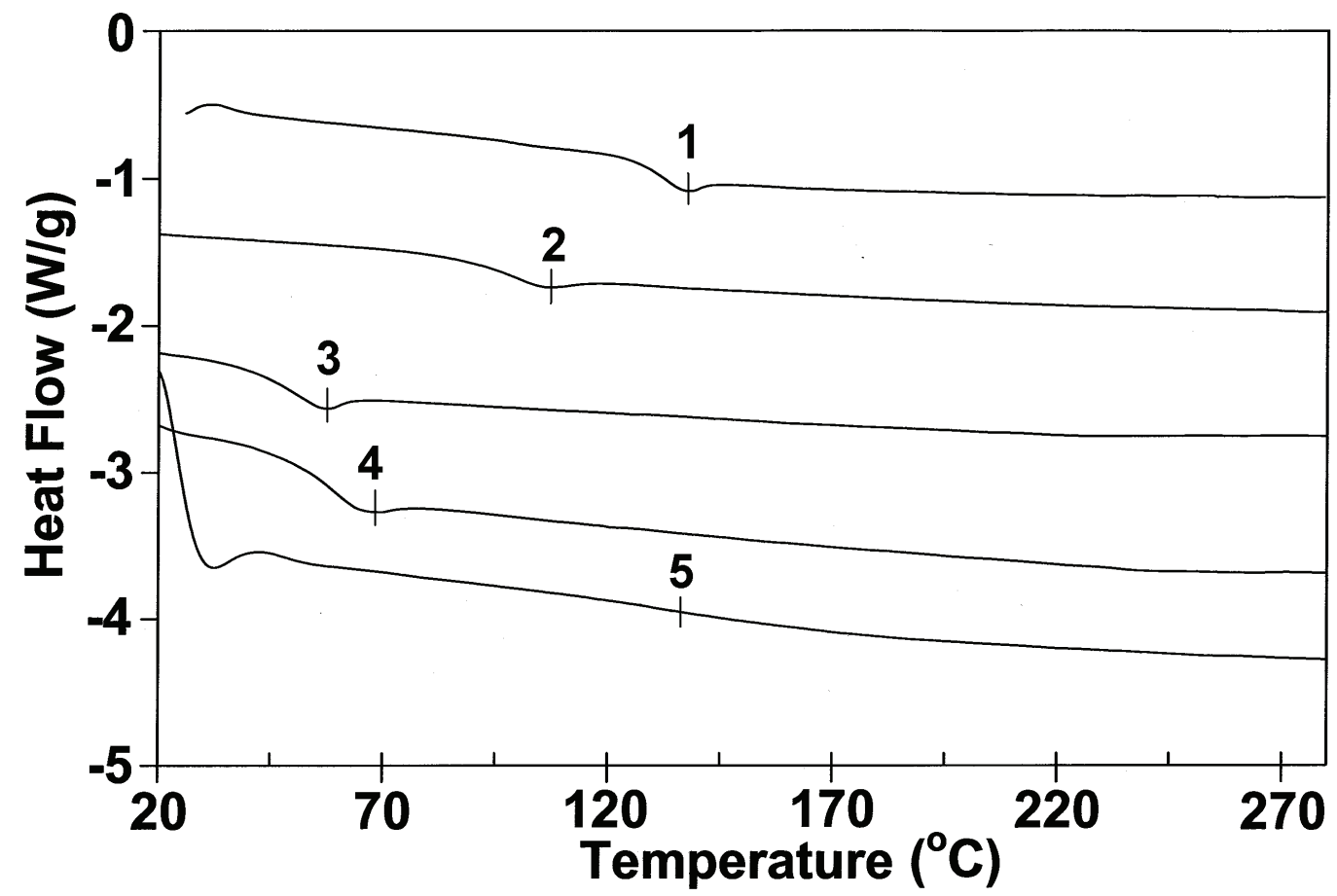

Figure S1. DSC thermograms of $\mathbf{1 - 5}$ measured under nitrogen at a heating rate of $10{ }^{\circ} \mathrm{C} / \mathrm{min}$ (recorded during the second heating scan).

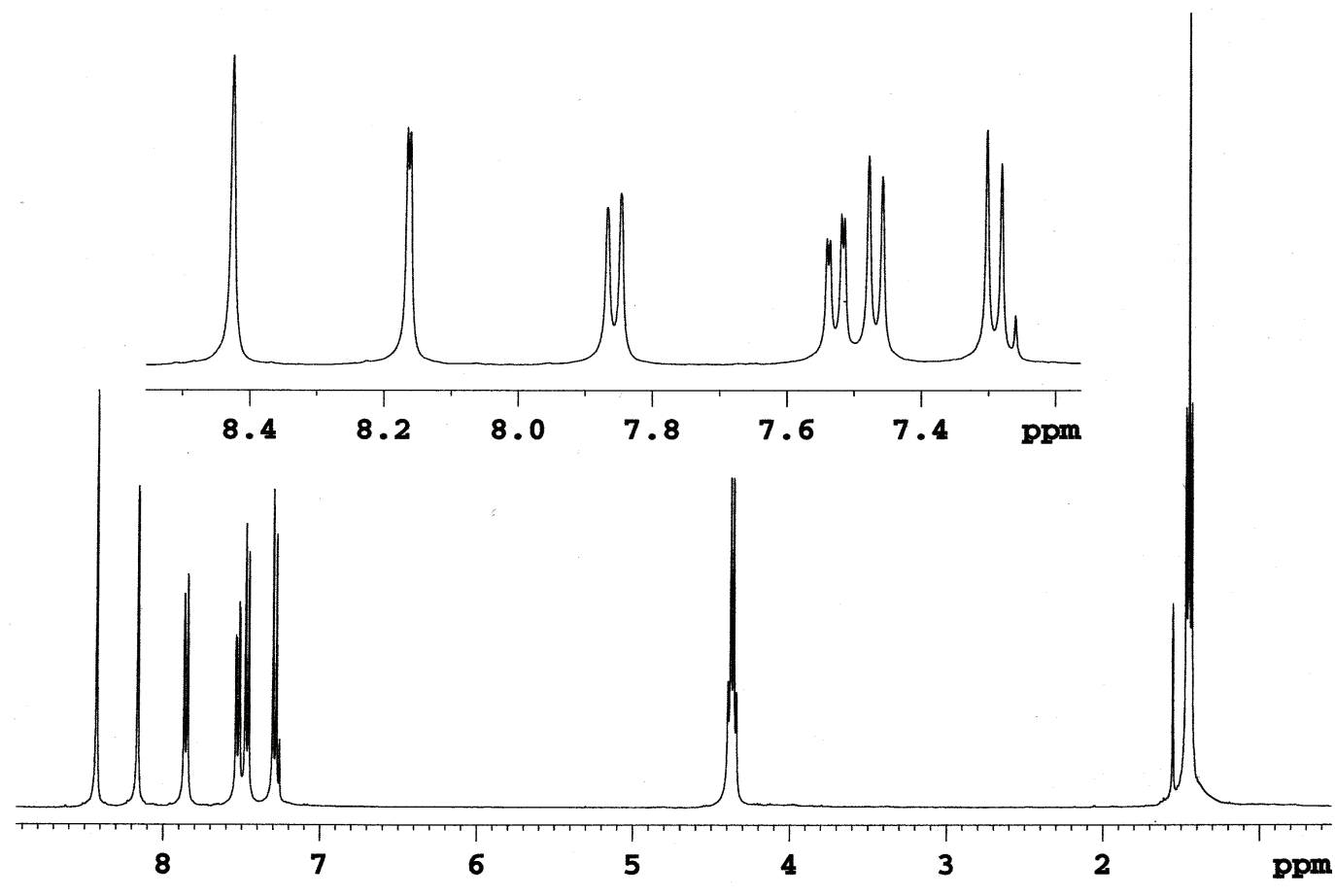

Figure S2. ${ }^{1} \mathrm{H}$ NMR spectrum of tetrahedral precursor 1 in $\mathrm{CDCl}_{3}$. 


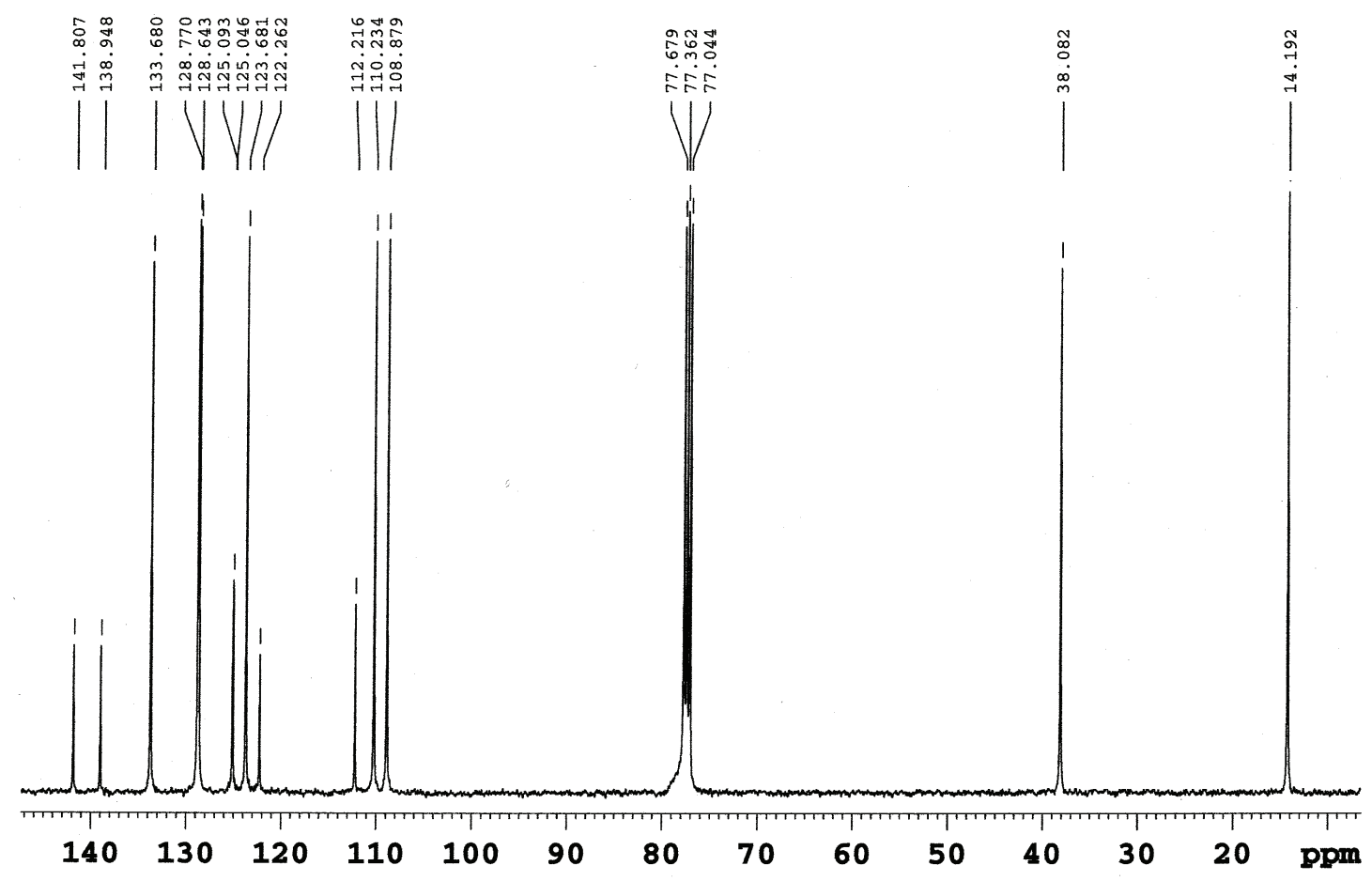

Figure S3. ${ }^{13} \mathrm{C}$ NMR spectrum of tetrahedral precursor 1 in $\mathrm{CDCl}_{3}$.

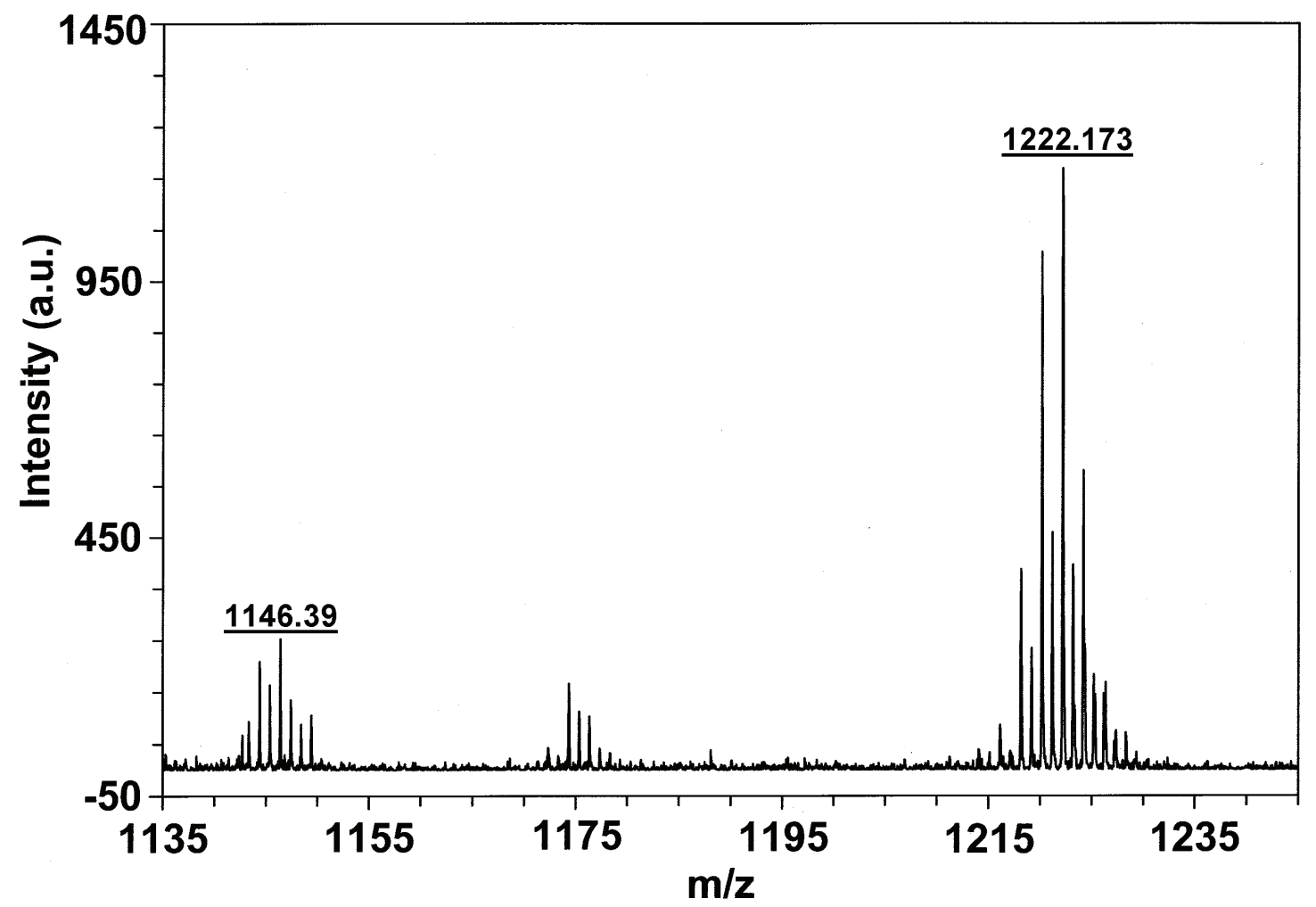

Figure S4. MALDI-TOF MS spectrum of tetrahedral precursor 1. 

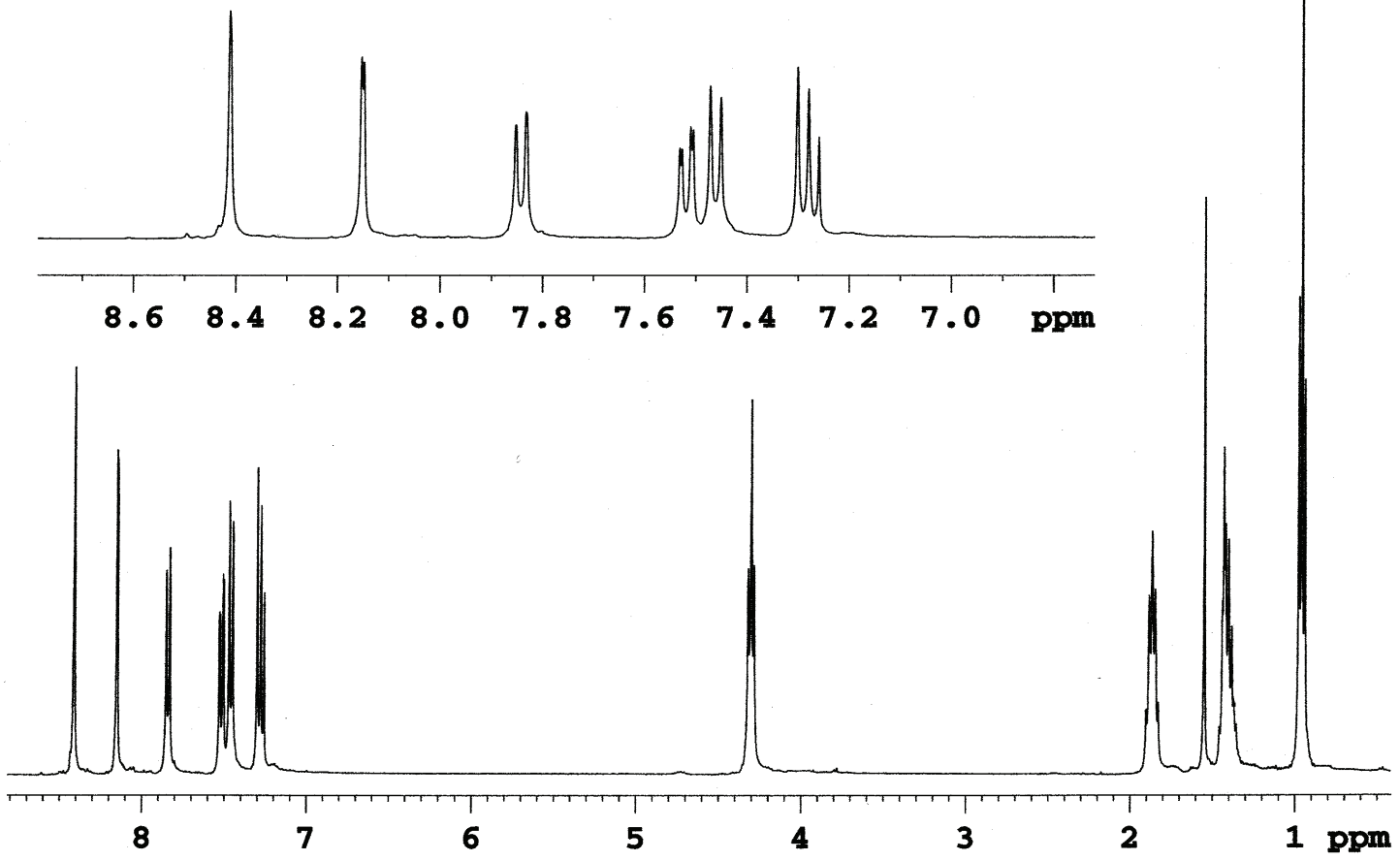

Figure S5. ${ }^{1} \mathrm{H}$ NMR spectrum of tetrahedral precursor 2 in $\mathrm{CDCl}_{3}$.

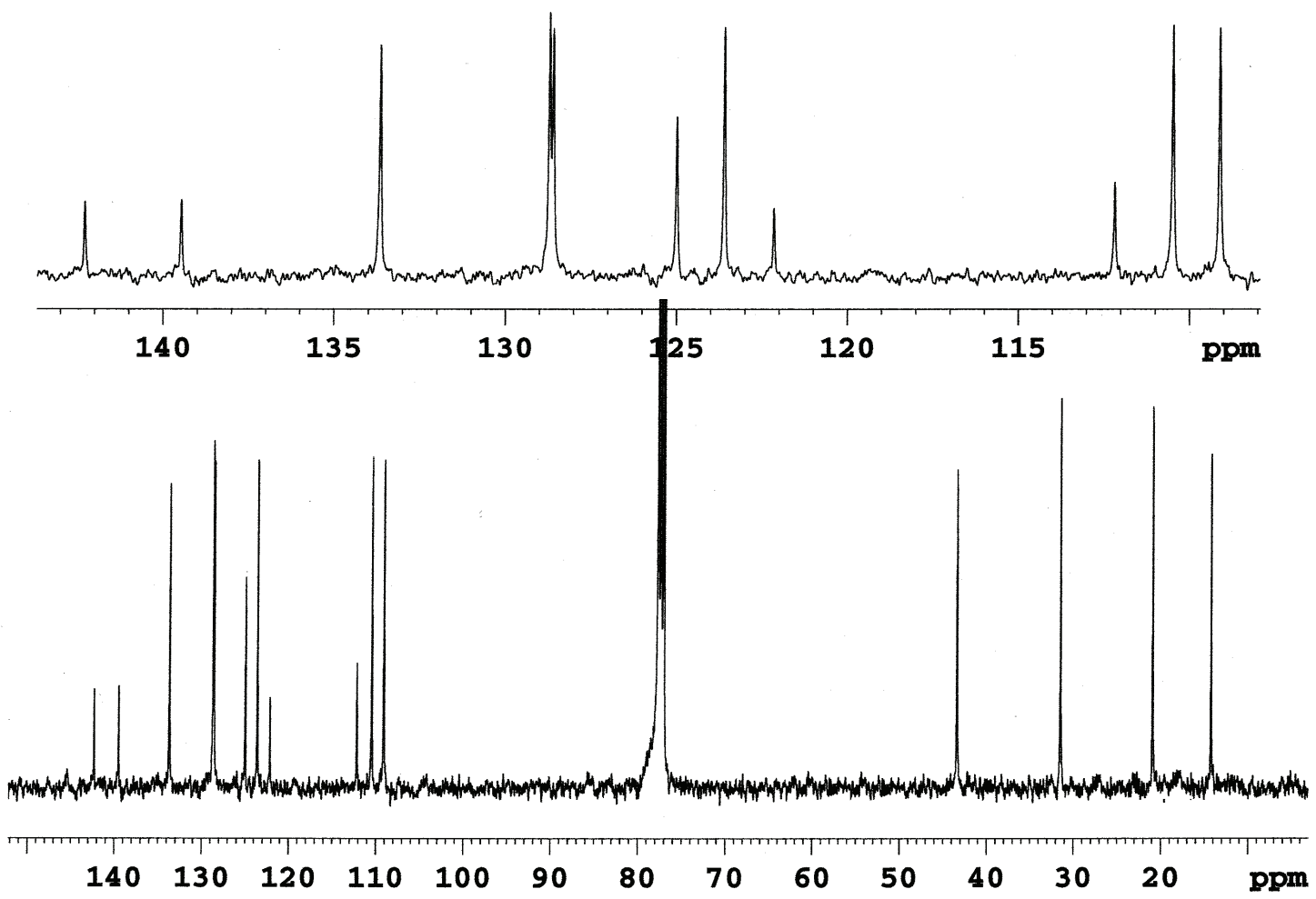

Figure S6. ${ }^{13} \mathrm{C}$ NMR spectrum of tetrahedral precursor 2 in $\mathrm{CDCl}_{3}$. 


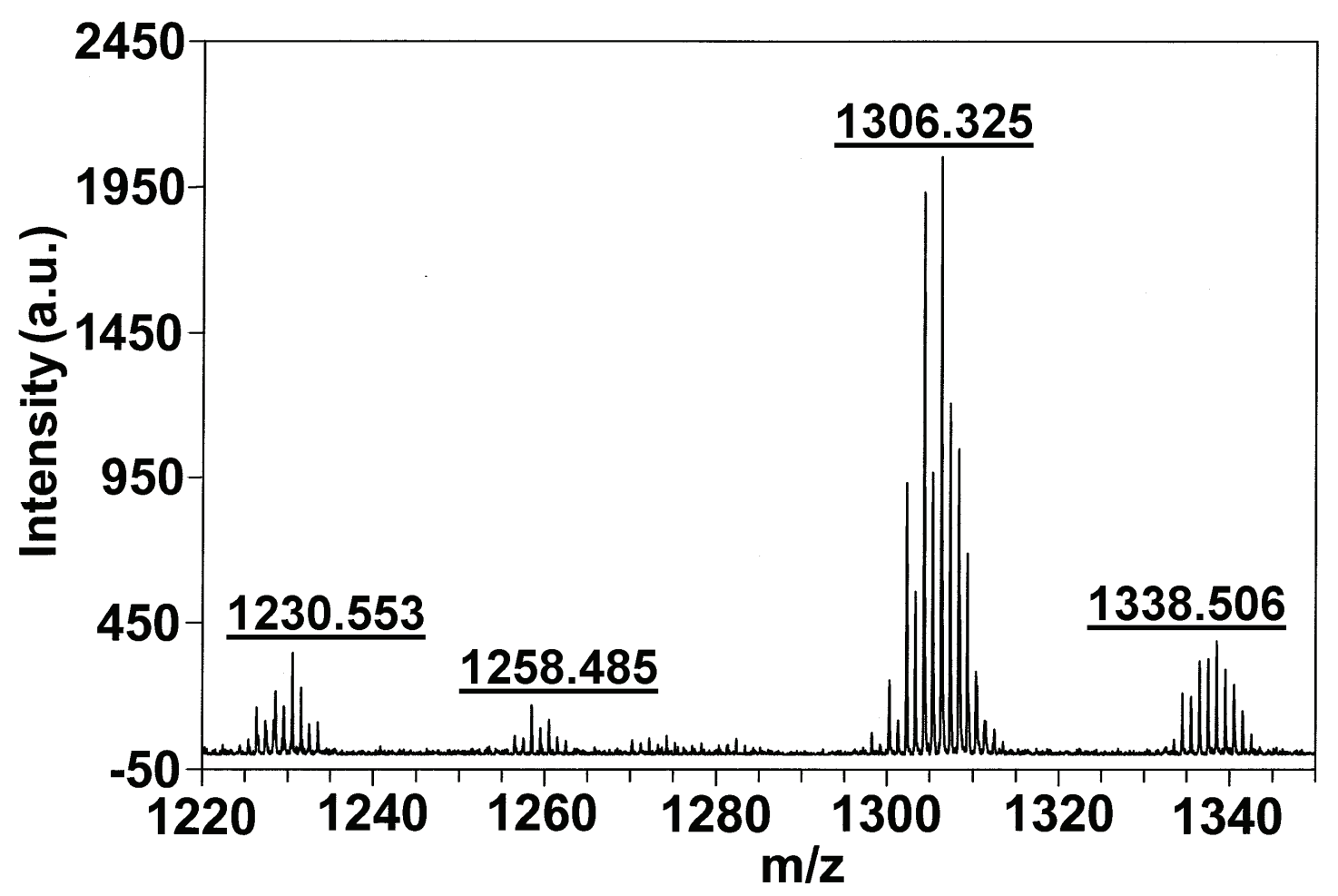

Figure S7. MALDI-TOF MS spectrum of tetrahedral precursor 2.

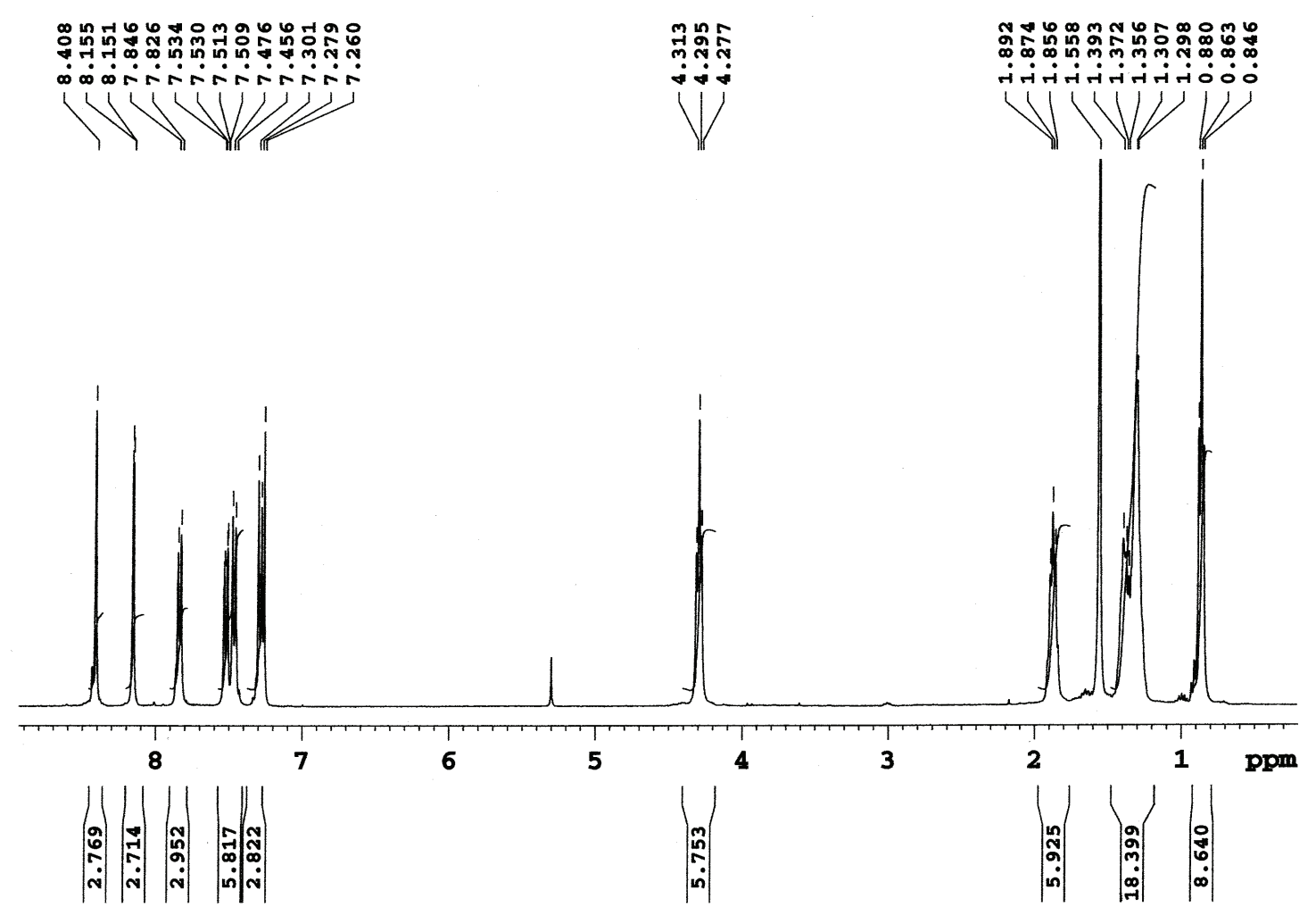

Figure S8. ${ }^{1} \mathrm{H}$ NMR spectrum of tetrahedral precursor 3 in $\mathrm{CDCl}_{3}$. 


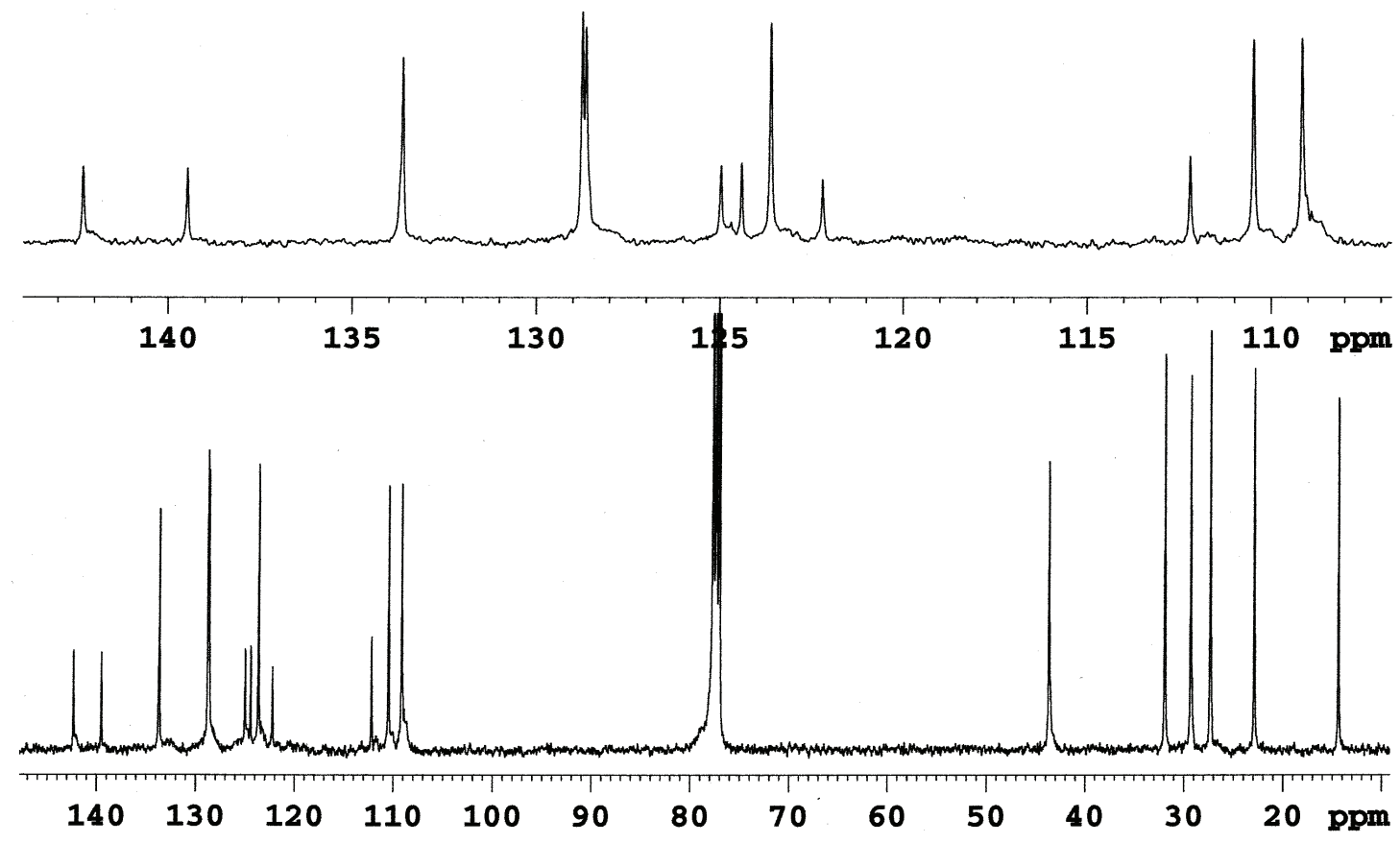

Figure S9. ${ }^{13} \mathrm{C}$ NMR spectrum of tetrahedral precursor 3 in $\mathrm{CDCl}_{3}$.

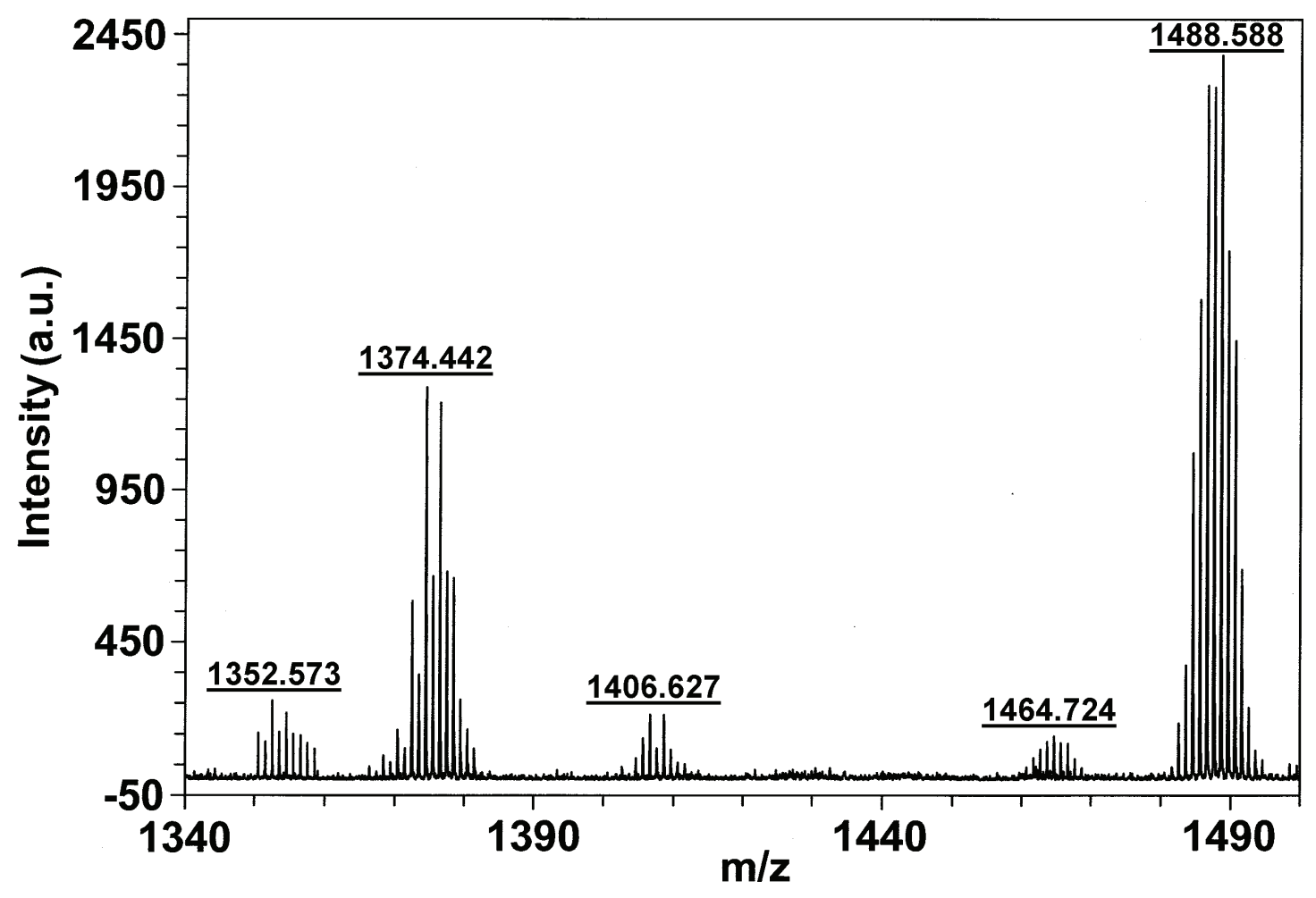

Figure S10. MALDI-TOF MS spectrum of tetrahedral precursor 3. 


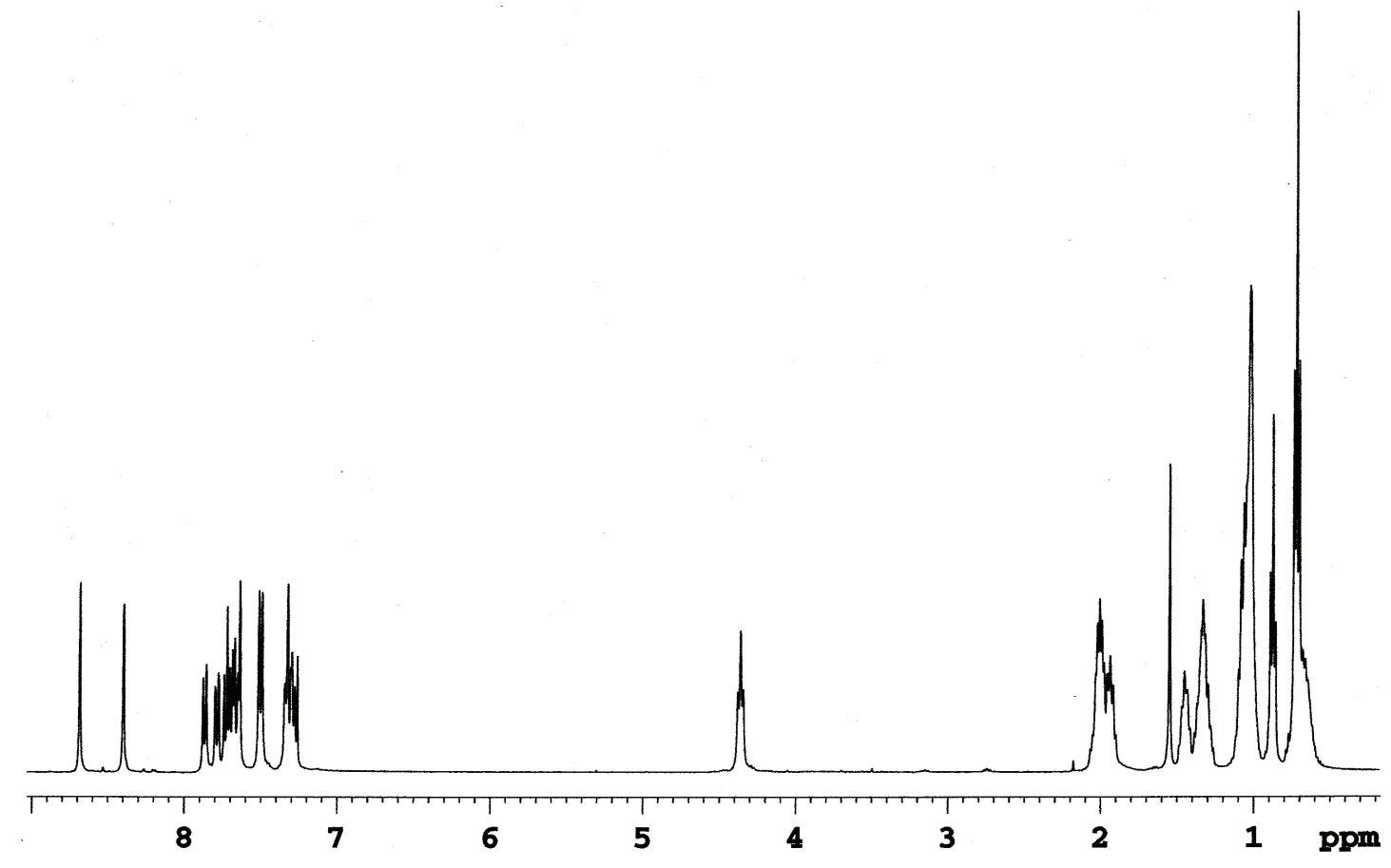

Figure S11. ${ }^{1} \mathrm{H}$ NMR spectrum of blue-light-emitting molecule 4 in $\mathrm{CDCl}_{3}$.

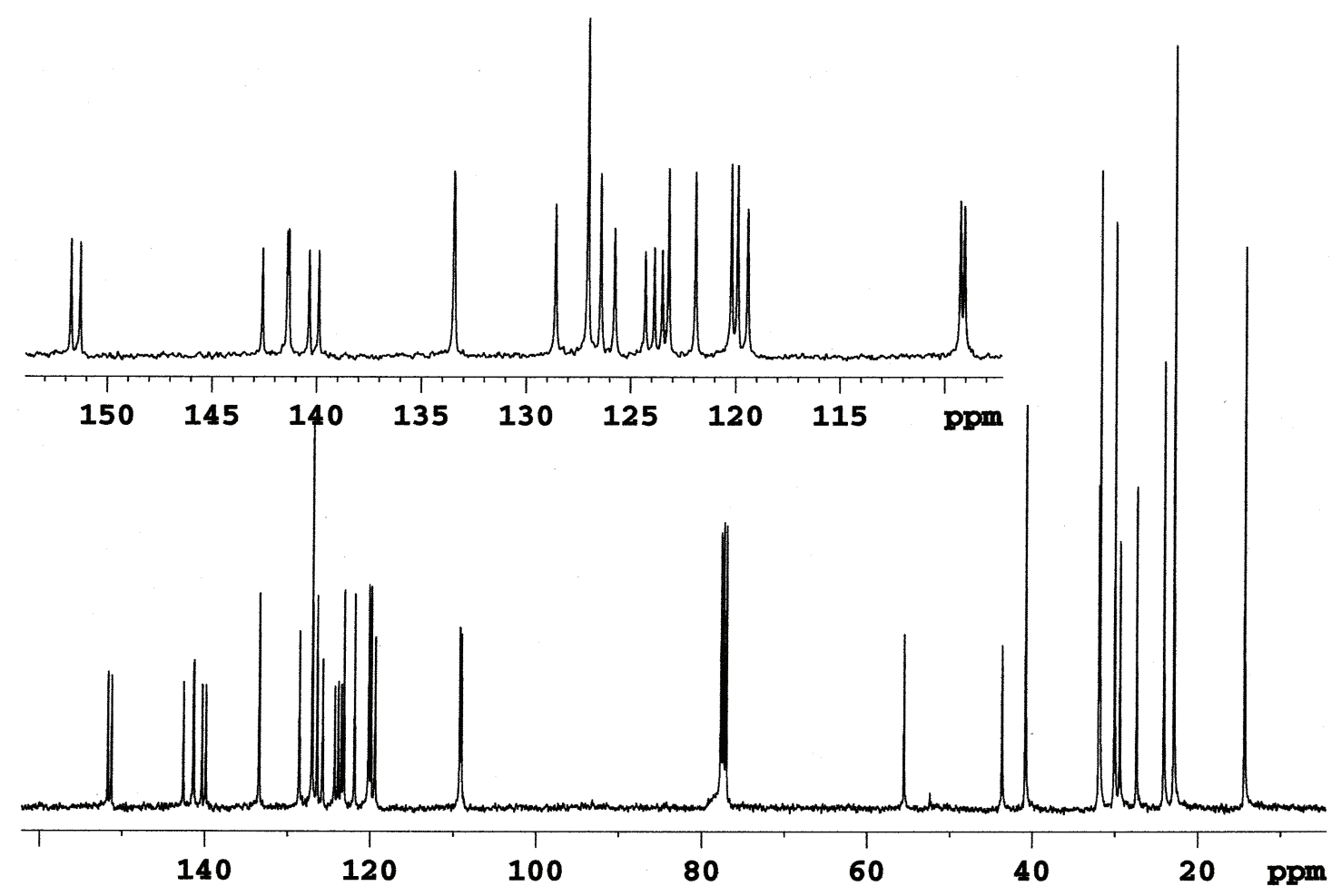

Figure S12. ${ }^{13} \mathrm{C}$ NMR spectrum of blue-light-emitting molecule 4 in $\mathrm{CDCl}_{3}$. 


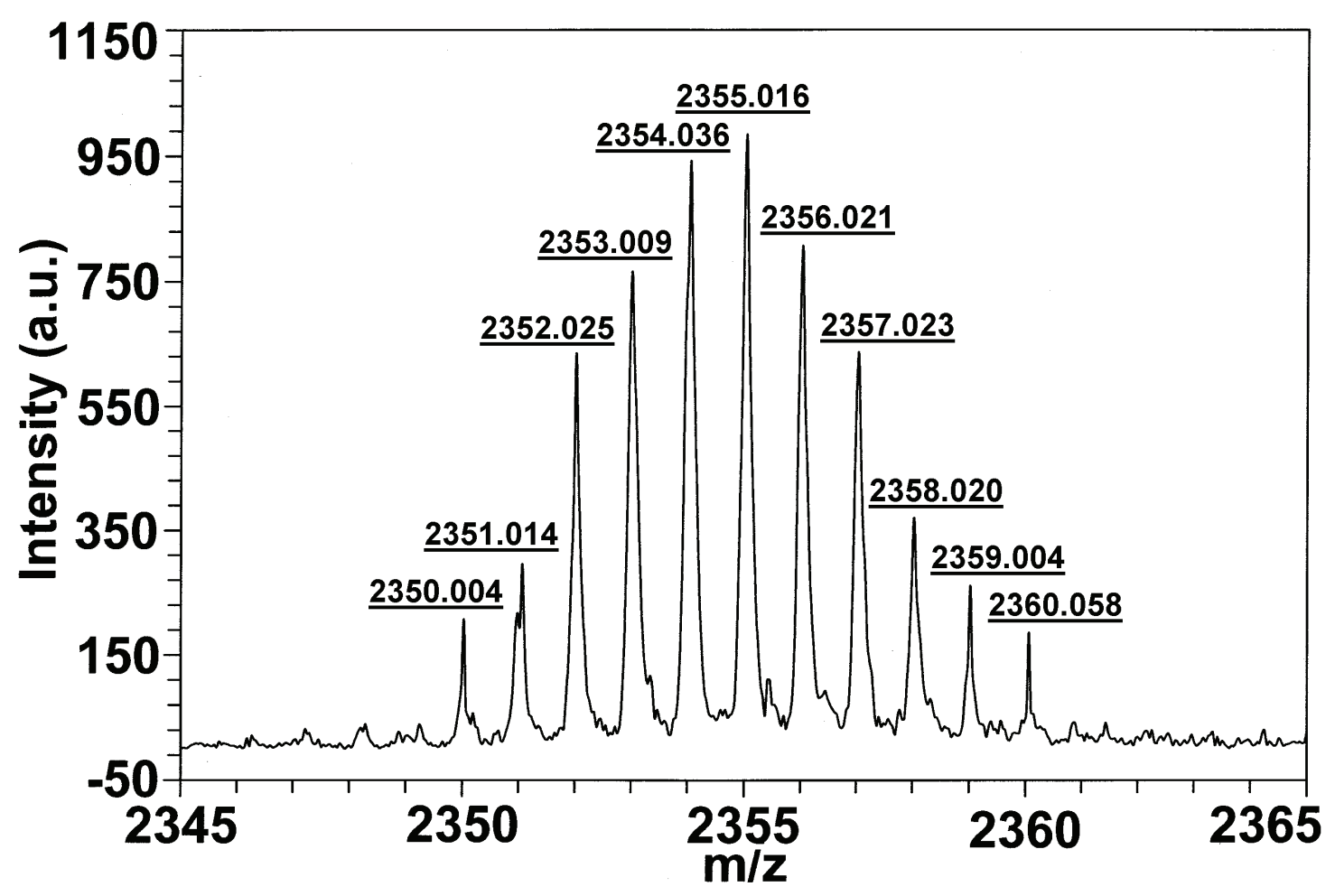

Figure S13. MALDI-TOF MS spectrum of blue-light-emitting molecule 4.

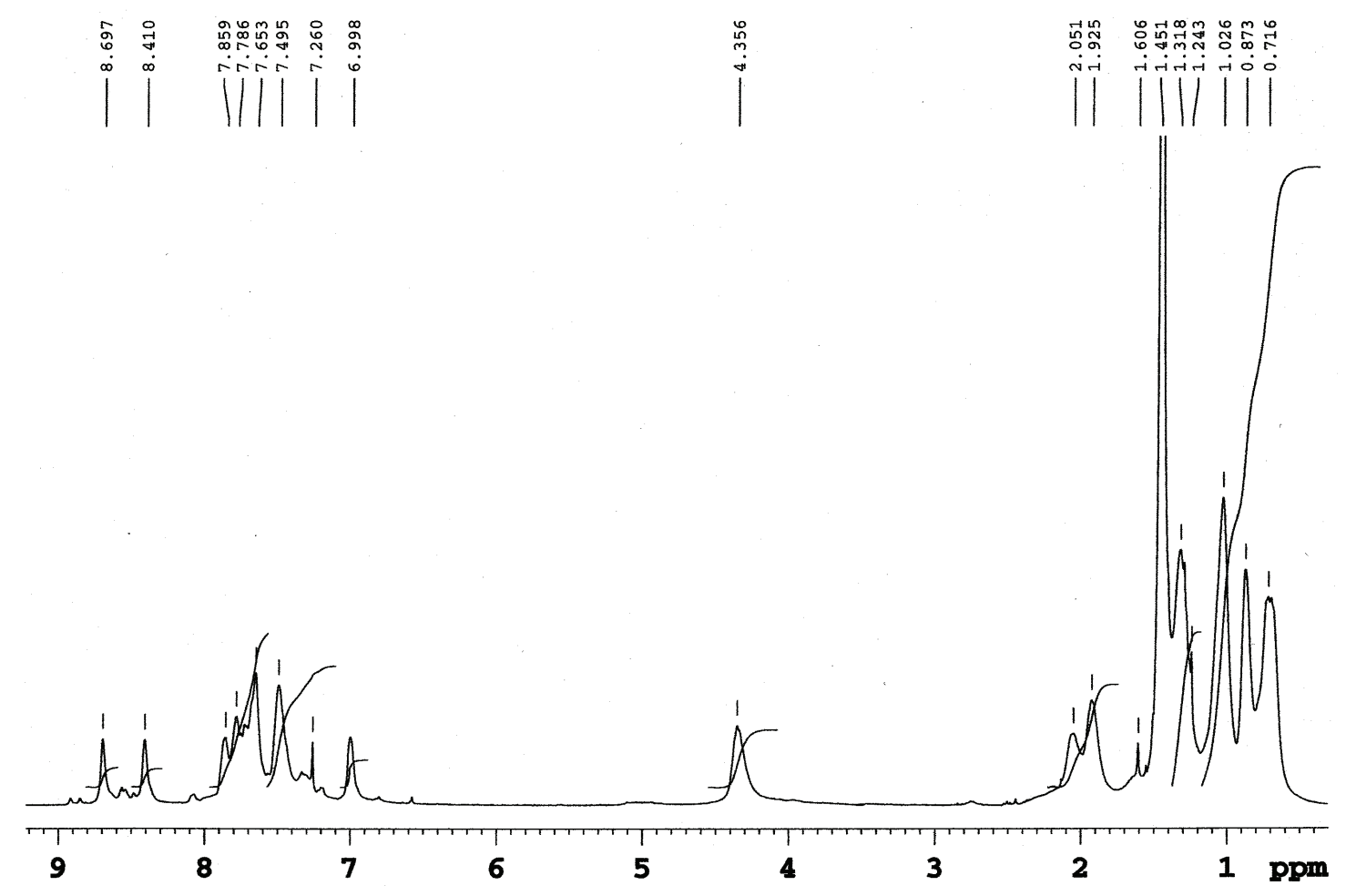

Figure S14. ${ }^{1} \mathrm{H}$ NMR spectrum of blue-light-emitting polymer 5 in $\mathrm{CDCl}_{3}$. 


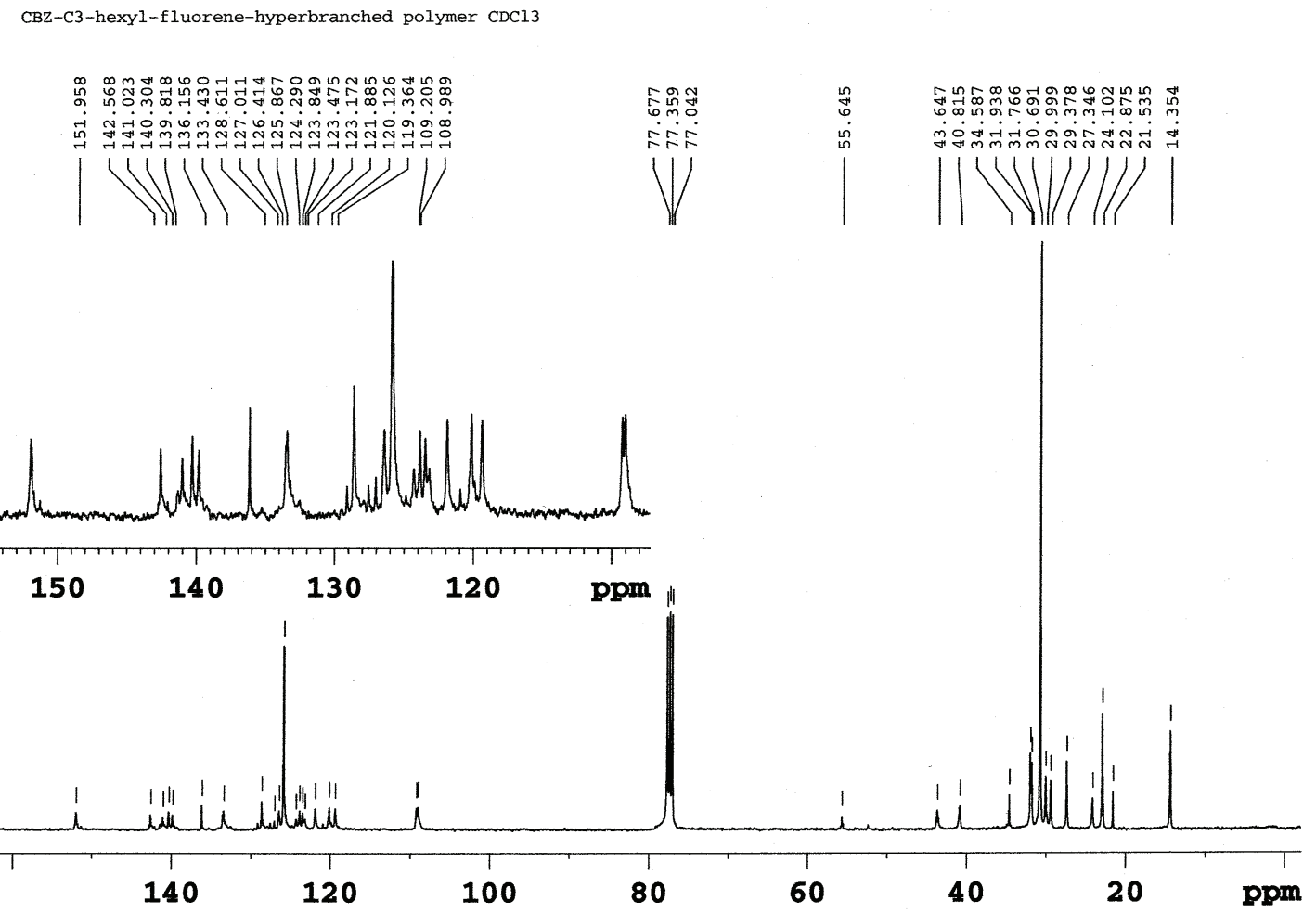

Figure S15. ${ }^{13} \mathrm{C}$ NMR spectrum of blue-light-emitting polymer 5 in $\mathrm{CDCl}_{3}$.

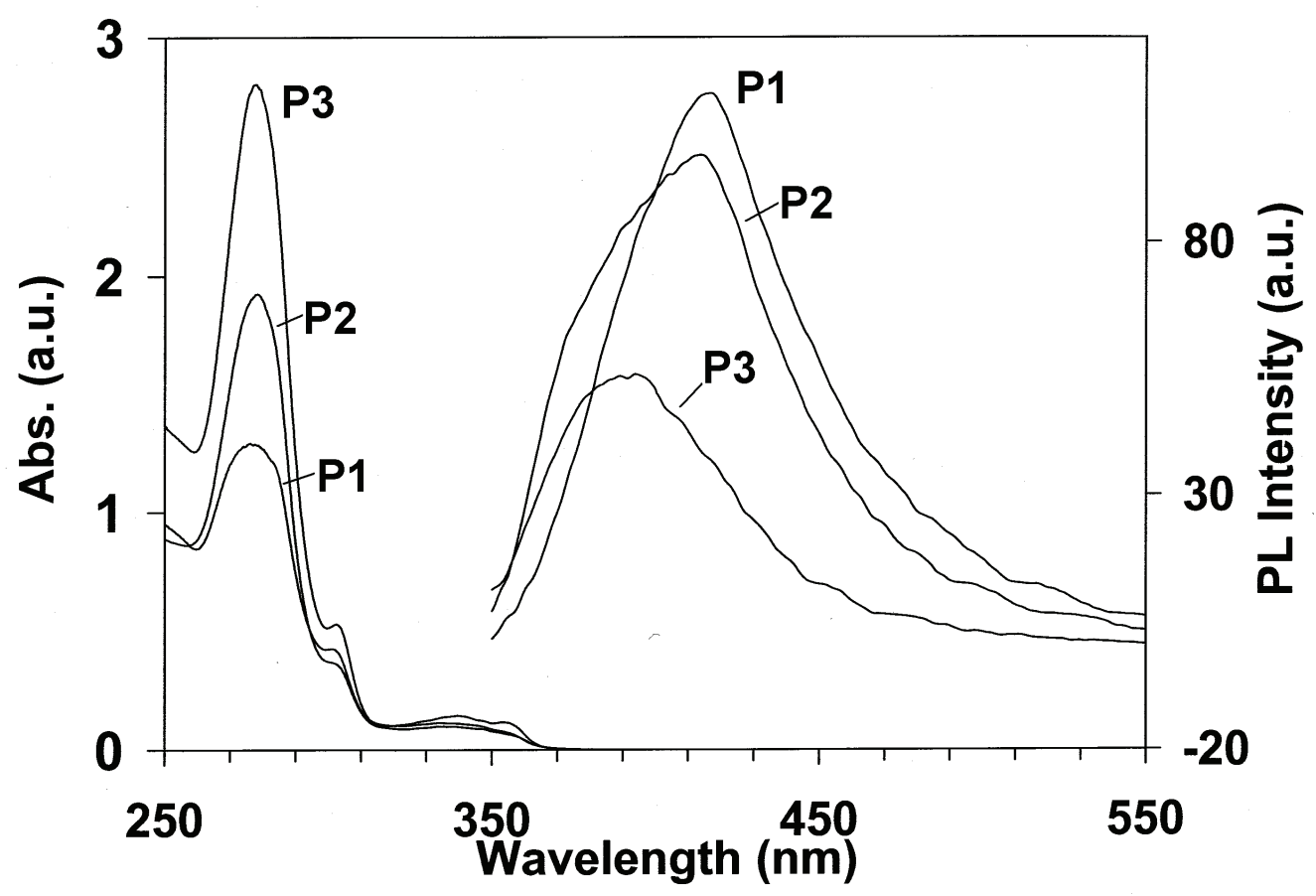

Figure S16. UV and PL emission spectra of P1-P3 in THF. 


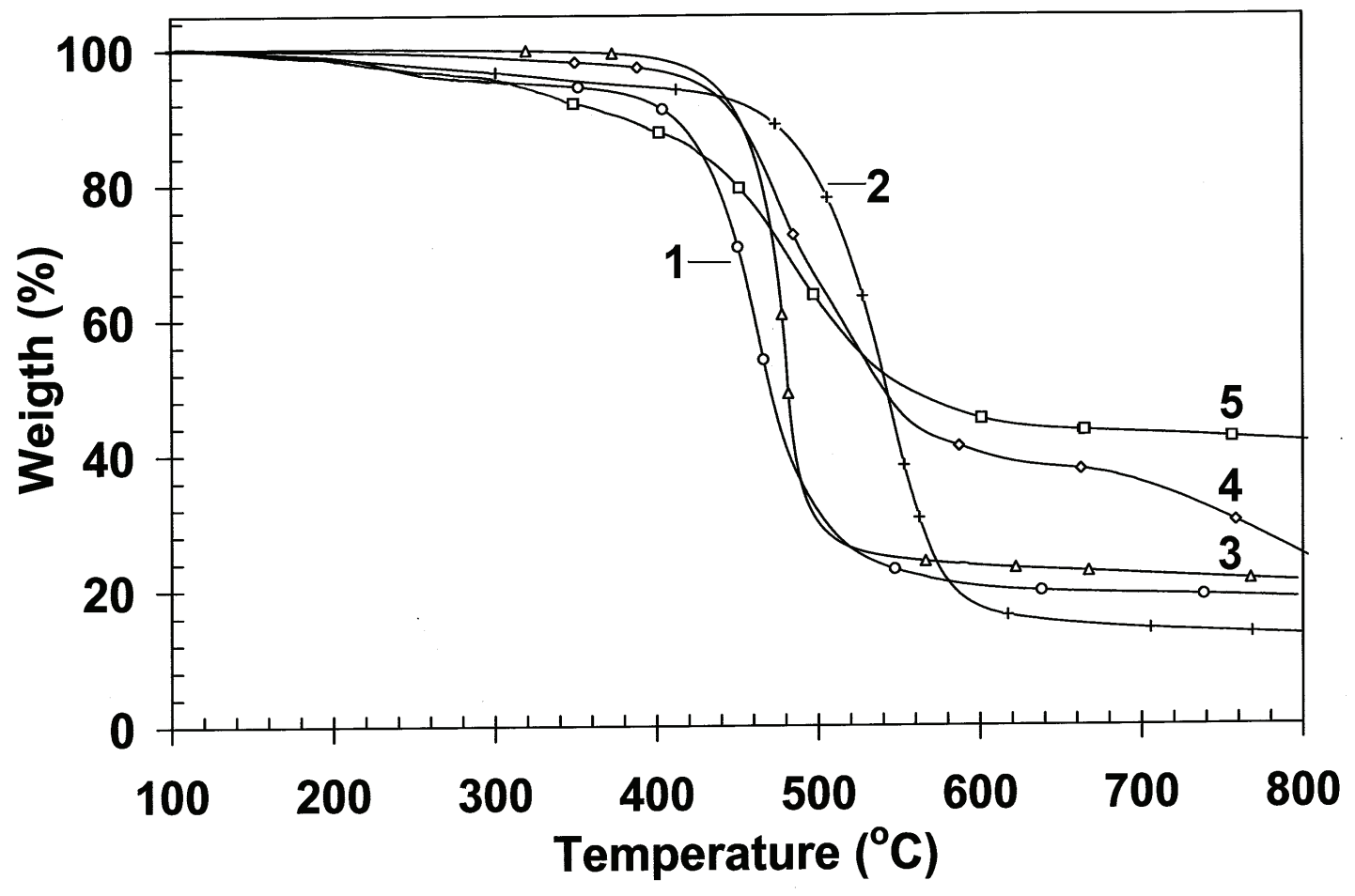

Figure S17. TGA thermograms of 1-5. The thermal analyses were carried out under nitrogen at a heating rate of $20^{\circ} \mathrm{C} / \mathrm{min}$. 